\section{Inserción posterior del fascículo superior del músculo pterigoideo externo en preparados anatómicos}

Posterior inserting of the upper fascicle of the lateral pterygoid muscle in anatomical preparations

\section{Resumen}

Este trabajo tuvo como objetivo determinar la prevalencia de inserción posterior del fascículo superior del músculo pterigoideo externo (Pteridoideo Lateral), en preparados anatómicos de cadáveres de adultos. Se seleccionó 4 cadáveres entre 25 y 60 años, se procedió a la disección hasta llegar a la fosa zigomática para exponer el músculo pterigoideo externo y los componentes de la ATM. Se identificó la relación de inserción posterior del fascículo superior del músculo pterigoideo externo con respecto al disco articular, cápsula articular y cóndilo mandibular, encontrándose que dicha inserción fue en el disco articular. Macroscópicamente no se observó inserción del fascículo superior del músculo pterigoideo externo a nivel del cuello de cóndilo.

Palabras claves: Músculos masticadores, ATM, cóndilo mandibular.

\section{Abstract}

This study aimed to determine the prevalence of posterior insertion of the superior fasciculus of the external pterygoid muscle (Lateral Pteridoideo) in anatomical preparations of adult corpses. 4 dead between 25 and 60 years was selected, was dissected up to the zygomatic fossa to expose the lateral pterygoid muscle and the components of the ATM. Subsequent insertion ratio upper lateral pterygoid muscle bundle with respect to the disc joint, joint capsule and condylar identified, being said insert was articular disk. Macroscopically, the upper bundle insertion lateral pterygoid muscle at condyle neck was observed.

Keywords: Masticatory muscles, TMJ, mandibular condyle.
Artículo OriginAL

\section{Justiniano Sotomayor Camayo', Jorge Eleodoro Villavicencio Gastelú́ ${ }^{2}$ Marieta Petkova- Gueorguieva1', Juana Delgadillo Ávila', Sergio Alvarado- Menacho ${ }^{3}$, Soledad Ana María Reyes Soto ${ }^{1}$, Carlos Humberto Campodónico Reátegui ${ }^{4}$, César Carranza López' ${ }^{1}$, Melissa Alisson Barra Hinostroza ${ }^{1}$}

\footnotetext{
1 Profesor del Departamento Académico de Ciencias Básicas FO-UNMSM.

2 Profesor del Departamento Académico Médico Quirúrgico FO-UNMSM.

3 Profesor del Departamento Académico de Estomatología Rehabilitadora FO-UNMSM.

4 Profesor del Departamento Académico de Estomatología Preventiva y Social FO-UNMSM.
}

\author{
Correspondencia: \\ Mg. Justiniano Sotomayor Camayo \\ Departamento de Ciencias Básicas de la Facultad \\ de Odontología de la Universidad Nacional Ma- \\ yor de San Marcos \\ Correo electrónico: justinianosc@hotmail.com
}

Fecha de recepción: 16-04-2013

Fecha de aceptación: 23-08-2013

\section{Introducción}

La articulación temporomandibular (ATM) ha sido tema de considerable interés en la investigación científica durante muchos años. Es sin duda una de las estructuras del sistema estomatognático más complejas, donde se pueden producir varios estados patológicos, cuyo correcto diagnóstico y tratamiento frecuentemente no son evidentes, ni fácilmente detectables.

Por lo tanto, es importante tener un conocimiento detallado de los componenetes de la ATM y en particular de la anatomía e inserción posterior del fascículo superior del músculo pterigoideo externo con el disco articular, ya que este tiene una gran importancia en la fisiología articulas y en los desórdenes temporomandibulares ${ }^{1}$.

La anatomía de la ATM ha sido objeto de debate no solo por su papel en la etiología del dolor articular, sino también por el hecho de que el haz superior del Pterigoideo externo (Pterigoideo Lateral -PL-) se encuentra insertado al disco o al cóndilo de la mandíbula y contribuye al desplazamiento del disco y al dolor articular. ${ }^{2,3,4,5}$
Mc Devitt ${ }^{3}$ y Kaplan ${ }^{4}$, pensaron que las fibras superiores del PL se insertaban en el disco y la cápsula, especialmente en la parte media, mientras que las fibras inferiores se insertaban en el cuello del cóndilo. Sin embargo, existen diferencias de opiniones sobre esto: unos piensan que no se insertan al disco ${ }^{7,8}$, pero una pequeña porción de fibras se encuentran insertadas en la parte anterior de la cápsula y por ende están insertadas directa e indirectamente al cóndilo y no al disco por si misma. Otros señalan que el PL se encuentra directamente insertado al disco y al cóndilo ${ }^{1,9}$. También se cree que solo esta insertado en 
el borde anterior de la cápsula y el dis$\mathrm{Co}^{10}$. Todos los investigadores están de acuerdo sobre la inserción anterior del PL al periostio de la fosa infratemporal adyacente al ala mayor del esfenoides. El porque existe esta confusión no esta claro, ya que los estudios involucran tanto exámenes macroscopicos como microscópicos del músculo en relación a la articulación.

Con respecto a lo anteriormente mencionado, en 1995 Heylings et $\mathrm{al}^{2}$ realizaron un estudio cuyo propósito fué determinar la relación anatómica entre el PL y el disco, y descubrir si el músculo se insertava directamente en la cápsula y el cóndilo de la mandíbula, además se pretendía determinar como se ha desarrollado la confusión en la literatura. Para llevar a cabo esta investigación se emplearon 16 muestras de cadáveres de individuos entre 60 a 90 años, 11 de esas muestras fueron edéntulos mientras que el resto fueron edéntulos parciales; el arco zigomático se removió en todos los casos y se secciono la rama de la mandíbula durante la disección inicial. Los resultados del estudio demostraron que solo una parte de la porción superior del músculo se inserta en la porción anterior de la cápsula, la cual a su vez se encuentra firmemente insertada al disco dando la impresión de que el músculo y el disco están directamente conectados. En todas las muestras se observo que el PL se inserta en la porción antero-medial de la cápsula, pero presentaban grados variables de inserción a la porción lateral de esta estructura. La parte restante del PL se inserta al cóndilo mandibular.

Tener conocimiento detallado de la anatomía del PL es importante, tiene especial relevancia tanto en su fisiología como a nivel de los trastornos articulares ${ }^{11}$, sin embargo, una revisión preliminar de la literatura reveló la falta de consenso sobre la anatomía de este músculo. De acuerdo con Honee ${ }^{10}$, el PL está formado por una porción superior y una inferior, mientras que otros investigadores como Grant ${ }^{12}$ no identificaron partes separadas. Por otro lado, la configuración anatómica de la inserción músculo-disco es debatible. Honee $^{10}$ y Porter $^{3}$ señalaron que el PL se encuentra insertado al disco, mientras que otros 36 investigadores, encontraron que solo una pequeña fracción de fibras se unía; sin embargo, Pinkert (mencionado por Heylings en su estudio $^{2}$ ) encontró una septa fibrosa separando al músculo del disco.

La función del PL es controversial: White $^{13}$ señalo que el disco se movía activamente debido a la contracción de este músculo, mientras que Findlay y Osborn ${ }^{14}$ encontraron solo movimientos secundarios del disco totalmente dependientes del cóndilo.

Pensamos que el fascículo superior del músculo pterigoideo externo se inserta a nivel posterior con mayor prevalencia en el disco articular ya que atraviesa la cápsula articular para llegar al mismo y tambien tiene inserción en el cóndilo.

La presente investigación, además del interés académico desde el punto de vista anatómico, contribuye directamente en el entendimiento de las inserciones del PL para el diagnóstico de las alteraciones de la ATM, e indirectamente en su influencia en la rehabilitación integral del paciente, específicamente de aquellos pacientes que presentan problemas articulares.

\section{Material y métodos}

Esta investigación es de tipo descriptivo, transversal y exploratorio. El estudio se realizó en preparados anatómicos de 8 cadáveres con edad entre 25 a 60 años. La muestra estuvo constituída por 16 preparados anatómicos correspondientes a la región de la ATM.

Pasos del estudio:

a. Se clasificó y enumeró el material cadavérico a disecar

b. Se procedió a disecar la región de la ATM, se retió el arco zigomático para ingresar a la fosa zigomática seccionándo la rama mandibular para exponer el fascículo superior del músculo pterigoideo externo y los componentes de la ATM: cápsula, disco articular, cóndilo temporal y cóndilo mandibular. Fig 1, 2 y 3

c. Se realizó el registro fotográfico

d. Se observó la relación del fascículo superior del músculo. pterigoideo externo con respecto al disco articular

e. Se registrarón los datos observados en las fichas de recolección de datos

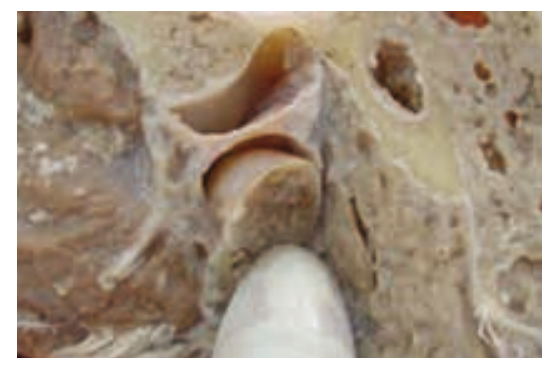

Fig. 1 Exposición del disco e inserción de PL al disco articular

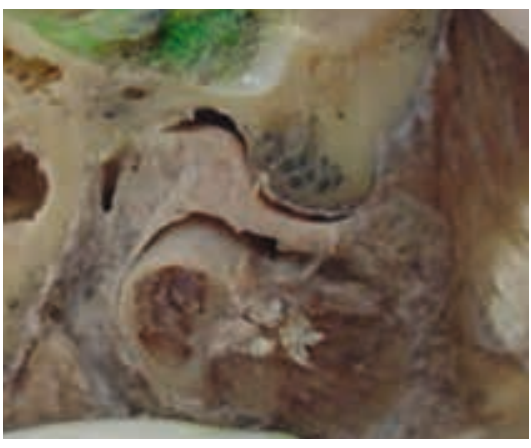

Fig. 2 Vista lateral del Disco Articular

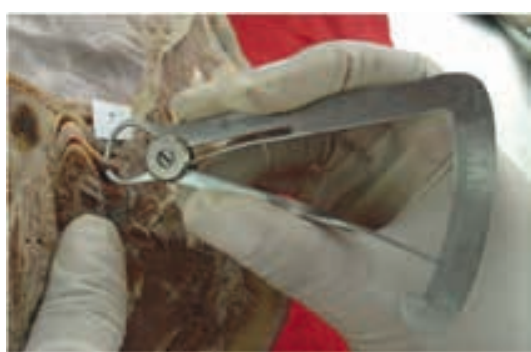

Fig. 3 Medición anterior de la inserción del PL vientre superior

\section{Resultados}

En el estudio macroscópico se halló que en todos los preparados anatómicos la inserción posterior del músculo pterigoideo externo fue en el disco articular, siendo la capsula (ligamento capsular) zona de paso, no encontrándose inserción a nivel del cóndilo.

También se registraron las medidas del grosor del músculo pterigoideo externo en sentido sagital, el espesor del disco articular en la parte media más delgada y la distancia zona bilaminar a conducto auditivo externo como puntos de referencia anatómico de posible utilidad en la interpretación de los aspectos clínicos a este nivel de la articulación temporomandibular.

En lo que respecta al grosor del músculo pterigoideo externo se encontró un promedio de $2.63 \mathrm{~mm}( \pm 0.54)$ entre los ocho preparados anatómicos.

Tabla 1. Grosor del músculo pterigoideo externo

MEDIA

DESVIACIÓN ESTÁNDAR

MEDIANA

MÍNIMO

MÁXIMO

RANGO INTERCUARTÍLICO 1.1 
Gráfico 1. Espesor del músculo pterigoideo externo (dirección sagital)

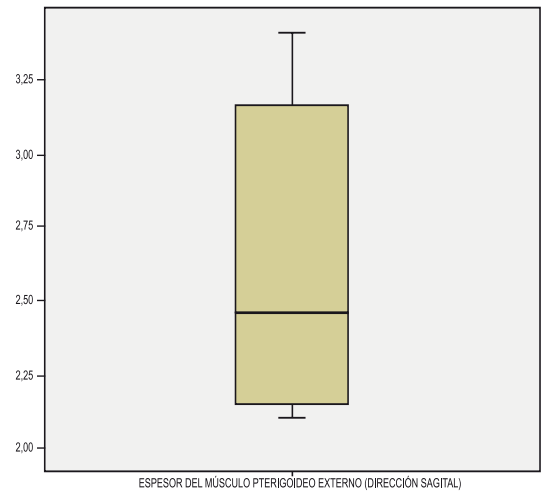

Gráfico 2. Espesor del disco (parte media más delgada)

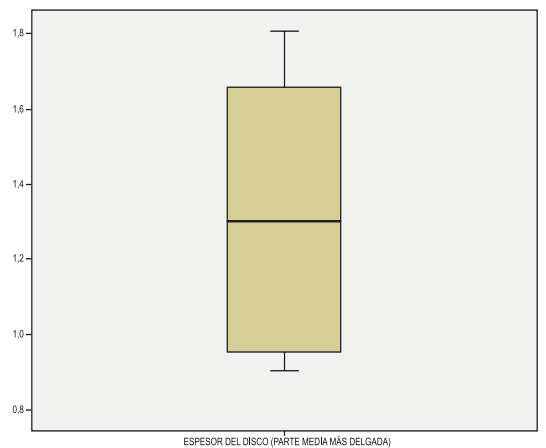

Gráfico 3. Distancia zona bilaminarconducto auditivo externo

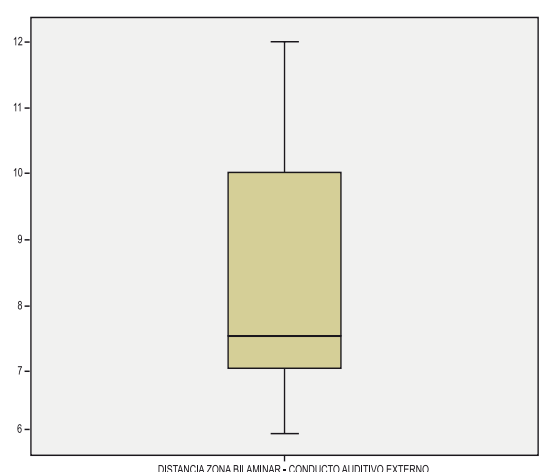

\section{Discusión}

Los resultados del estudio anatómico sobre la inserción posterior del músculo pterigoideo externo fue en el disco articular, no encontrándose inserción a nivel del cóndilo. Mientras que Mc Devitt y Kaplan ${ }^{6}$, pensaron que las fibras superiores del PL se insertaban en el disco y la cápsula, especialmente en la parte media, mientras que las fibras inferiores se insertaban en el cuello del cóndilo. Lo que podemos decir que el estudio realizado, difiere de los resul-

El espesor promedio del disco en la parte media más delgada fue de $1.31 \mathrm{~mm}$ $( \pm 0.36)$. Así como se puede observar en Tabla 2 .

Tabla 2 . Espesor del disco en la parte media

\begin{tabular}{lc}
\hline MEDIA & 1.31 \\
DESVIACIÓN ESTÁNDAR & 0.36 \\
MEDIANA & 1.3 \\
MÍNIMO & 0.9 \\
MÁXIMO & 1.8 \\
RANGO INTERCUARTÍLICO & 0.75
\end{tabular}

La distancia promedio de la zona bilaminar - conducto auditivo externo fue de $8.38 \mathrm{~mm}( \pm 2.13)$ Tabla 3 .

Tabla 3 . Distancia zona bilaminar-conducto auditivo externo

\begin{tabular}{ll} 
MEDIA & 8.38 \\
DESVIACIÓN ESTÁNDAR & 2.134 \\
MEDIANA & 7.5 \\
MÍNIMO & 6 \\
MÁXIMO & 12 \\
RANGO INTERCUARTÍLICO & 3.5 \\
\hline
\end{tabular}

La mediana fue de $7.5 \mathrm{~mm}$, el valor mínimo fue de $6 \mathrm{~mm}$ y el máximo de $12 \mathrm{~mm}$ La distribución de éstos datos y los anteriores fue normal.

Tabla 4. Distribución de los datos.

\begin{tabular}{lllllll}
\hline & \multicolumn{3}{l}{ Kolmogorov-Smirnov(a) } & Shapiro-Wilk & & \\
& Estadístico & gl & Sig. & Estadístico & gl & Sig. \\
$\begin{array}{l}\text { Espesor del músculo pterigoi- } \\
\text { deo externo (dirección sagital) }\end{array}$ & 0,23 & 8 & 0,2 & 0,86 & 8 & 0,121 \\
$\begin{array}{l}\text { Espesor del disco (parte } \\
\text { media más delgada) }\end{array}$ & 0,18 & 8 & 0,2 & 0,90 & 8 & 0,269 \\
$\begin{array}{l}\text { Distancia zona bilaminar- } \\
\text { conducto auditivo externo }\end{array}$ & 0,24 & 8 & 0,194 & 0,88 & 8 & 0,197 \\
\hline
\end{tabular}

tados de estos autores porque ellos encontraron hasta triple inserción, en la cápsula, disco y cuello del cóndilo.

En 1995 Heylings et al. ${ }^{2}$ realizaron un estudio cuyo propósito fue determinar la relación anatómica entre el PL y el disco. Para llevar a cabo esta investigación se emplearon 16 muestras de cadáveres de individuos de 60 a 90 años. Los resultados del estudio demostraron que solo una parte de la porción superior del músculo se insertó en la porción anterior de la cápsula, la cual a su vez se encuentra firmemente insertada al disco dando la impresión de que el músculo y el disco están directamente conectados. Como vemos el también encontró doble inserción: cápsula del cóndilo y disco, solo identificó que el disco estaba directamente conectado. Nuestro estudio coincide con el estudio realizado por estos autores.

Nuestros resultados coinciden con Porter quien señaló que el PL se encuentra insertado al disco, mientras que Pinkert (mencionado por Heylings en su estu- 
dio $^{2}$ ) encontró que solo una pequeña fracción de fibras se unía separando al músculo del disco.

Podemos decir que la discrepancia sigue existiendo entre los diferentes autores en relación a la inserción del cóndilo, pero nuestro estudio si encontró coincidencia con algunos investigadores.

\section{Conclusiones}

La inserción posterior del músculo pterigoideo externo superior, en éste estudio se ubicó directamente en la parte anterior del disco articular.

El fascículo superior del músculo pterigoideo externo para insertarse en el disco atraviesa la cápsula articular pero no se inserta a nivel del cuello de cóndilo.

Referente al grosor del músculo pterigoideo externo se encontró un promedio delgado entre los ocho preparados anatómicos.

El espesor promedio del disco en la parte media más delgada, fue menor en un milímetro que el grosor del músculo pterigoideo externo fascículo superior en su inserción posterior, no siendo relevante la distancia de zona bilaminar al conducto auditivo externo.

Se recomienda realizar un estudio según grupos étnicos y también un estudio microscópico.

\section{Referencias bibliográficas}

1. Schmolke C. The relationship between the temporomandibular joint capsule, articular disc and jaw muscles. J Anat. 1994;184(2):335-345.
2. Heylings DJ, Nielsen IL, McNeill C. Lateral pterygoid muscle and the temporomandibular disc. J Orofacial Pain. 1999;9(1): 9-16.

3. McDevitt W. Functional anatomy of the masticatory system. London: Butterworth; 1989. 560-564 p.

4. Murray MG, Phanachet I. The human lateral pterygoid muscle: A review of some experimental aspects ans possible clinical relevance. Aust Dent J. 2004; 49(1): 2-8.

5. Phanachet I, Whittle T. Functional heterogeneity in the superior head of the human lateral pterygoid. J Dent Res. 2003; 82(2):106-11.

6. Kaplan A, Assael L. Temporomandibular disorders: Diagnosis and treatment. Philadelphia: WB Saunders; 1991. 226-235 p.

7. Mahan PE, Wilkinson TM, Gibbs $\mathrm{CH}$, Mauderli A, Brannon LS.. Superior and inferior bellies of the lateral pterygoid muscle EMG activity at basic jaw positions. J Prosthet Dent. 1983; 50(5):710-8.

8. Wilkinson T, Chan E. The anatomic relationship of the insertion of the superior lateral pterygoid muscle in the human temporomandibular joint of the human cadavers. Aus Dent J. 1989; 34(4):315-22.

9. Le toux G, Duval J. The human temporomandibular joint: Current anatomic and phycologic status. Surg Radiol Anat. 1989;11(4):283-8.

10. Honee G. The anatomy of the lateral pterygoid muscle. Acta Morphol Neerl Scand. 1972;10(4):331-335.
11. Burdi A. Morphogenesis. In: Sarnat B, Laskin D. The temporomandibular joint: A biological basis for clinical practice. ED 4. Philadelphia: WB Saunders;1992.36-47p.

12. Grant J. A method of anatomy descriptive and deductive. Baltimore: Williams and Wilkins; 1958. 704 p.

13. White L. The lateral pterygoid muscle: Fact and fictions. J Clin Orthod. 1985;19(8):584-587.

14. Osborn J. The disc of the human temporomandibular joint: Design, function and failure. J Oral Rehabil. 1985;12(4):279-93.

\section{Agradecimiento}

Agradecemos al Vicerrectorado de Investigación de la UNMSM por el apoyo económico para la ejecución de este estudio 